\title{
A Consistent Binormalized Data-Reusing LMS Algorithm for Noisy FIR Models
}

\author{
Byung Hoon Kang, Nam Kyu Kwon, Hyon-Taek Choi, and Poo Gyeon Park
}

\begin{abstract}
This paper proposes a consistent binormalized data-reusing least mean square (LMS) algorithm for identifying finite impulse response models whose input and output are corrupted by additive white noise. The proposed algorithm exploits the stochastic properties of the noisy input to compensate a bias of estimation which is occurred by input noise. Furthermore, by reusing the input signal, the algorithm overcomes a decline of convergence performance with highly correlated input signal. The experimental results show that the proposed algorithm achieves consistent estimation with noisy input signal. Furthermore, the proposed algorithm gets faster convergence rate and smaller steady-state estimation errors than the ordinary consistent LMS algorithms when the input signal is highly correlated.
\end{abstract}

Index Terms-Normalized least mean square algorithm (nLMS), consistent estimation, input noise, bias compensation, FIR channel estimation.

\section{INTRODUCTION}

It is well known that the ordinary least squares method yields unbiased parameters estimates when the data matrix is known exactly but if the data matrix or input signal is corrupted by noise then the estimates are biased [1], [2], [3]. There are several approaches that to obtain an unbiased estimate including the total least squares method which uses the singular value decomposition of a matrix, and modified least squares method which is to remove the noise-induced bias [2], [3], [4]. However, these approaches need huge computational complexity, generally $\mathrm{O}\left(\mathrm{N}^{\wedge} 3\right)$. Therefore, the algorithm which can achieve unbiased estimation while it needs less computational complexity have been studied [5],

Manuscript received October 14, 2012; revised November 15, 2012. This research was supported by the MKE(The Ministry of Knowledge Economy), Korea, under the ITRC(Information Technology Research Center) support program supervised by the NIPA(National IT Industry Promotion Agency)" (NIPA-2012-(H0301-12-1003)) and NIPA-2012-(H0301-12-2002)). This research was supported by World Class University program funded by the Ministry of Education, Science and Technology through the National Research Foundation of Korea (R31-10100). This research was also supported by “"Development of technologies for an underwater robot based on artificial intelligence for highly sophisticated missions", which is supported by KORDI.

Byung Hoon Kang and Nam Gyu Kwon are with the Department of Electrical Engineering, Pohang University of Science and Technology, San 31, Hyojadong, Namgu, Pohang, Kyungbuk, 790-784, Korea (e-mail: anbabo@postech.ac.kr, kwunnam@postech.ac.kr).

Hyun-Taek Choi is with the Korea Ocean Research \& Development Institute, Korea (e-mail: htchoiphd@kordi.re.kr).

Poo Gyeon Park is with the Division of IT Convergence Engineering and the Department of Electrical Engineering, Pohang University of Science and Technology, San 31, Hyojadong, Namgu, Pohang, Kyungbuk, 790-784, Korea (e-mail: ppg@postech.ac.kr).
[6]. Recently, the least mean square (LMS) type algorithm getting consistent estimate have been proposed [7]. The LMS-type algorithm can obtain consistent solution with much less computation, but has relatively slow convergence rate, especially when correlated input signal is used.

By the way, it is reported that the convergence performance can be improved by reusing input data [1].

Particularly a binormalized data-reusing LMS (BNDR-LMS) algorithm achieved good convergence rate with small additional computation [8], [9].

Inspired by those researches, we propose a consistent binormalized data-reusing least mean square (LMS) algorithm for identifying finite impulse response models whose input and output are corrupted by additive white noise. The proposed algorithm exploits the stochastic properties of the noisy input to compensate a bias of estimation which is occurred by additive white noise. Furthermore, the algorithm reuses input signal to overcome a decline of convergence performance with highly correlated input signal. The proposed algorithm has smaller steady-state estimation errors than the ordinary normalized LMS or BNDR-LMS and gets fast convergence rate compared to the consistent normalized LMS proposed in [7].

This paper is organized as follows. In Section II, we states the system model we concern. The statement of the problem and proposed algorithm is provided in Section III. The experimental results and concluding remarks are given in Section IV and Section V, respectively.

\section{Preliminary: Signal Model}

The adaptive filter is used to estimate the unknown system with FIR vector $\mathbf{h} \in R^{M \times 1}$. The desired value $d(n)$ is represented as

$$
d(n)=\mathbf{u}^{T}(n) \mathbf{h}+v_{o}(n)
$$

where $\mathbf{u}(n)=[u(n), u(n-1), \ldots, u(n-M+1)]^{T}$ denotes the noise-free input vector and $v_{o}(n)$ denotes observation noise with variance $\sigma_{o}^{2}$. The noisy input vector of the adaptive filter is given by

$$
\tilde{\mathbf{u}}(n)=\mathbf{u}(n)+\mathbf{v}_{\mathbf{i}}(n)
$$

where $\mathbf{v}_{\mathbf{i}}(n)=\left[v_{i}(n), v_{i}(n-1), \ldots, v_{i}(n-M+1)\right]^{T}$.The following assumptions are introduced. 


$$
\begin{aligned}
& \frac{\lambda_{1}}{2}= \frac{\left(d(n)-\mathbf{u}^{T}(n) \mathbf{w}(n)\right) \mathbf{u}^{T}(n-1) \mathbf{u}(n-1)-\left(d(n-1)-\mathbf{u}^{T}(n-1) \mathbf{w}(n)\right) \mathbf{u}^{T}(n) \mathbf{u}(n-1)}{\mathbf{u}^{T}(n) \mathbf{u}(n) \mathbf{u}^{T}(n-1) \mathbf{u}(n-1)-\left(\mathbf{u}^{T}(n) \mathbf{u}(n-1)\right)^{2}+\epsilon}, \\
& \frac{\lambda_{2}}{2}=\frac{\left(d(n-1)-\mathbf{u}^{T}(n-1) \mathbf{w}(n)\right) \mathbf{u}^{T}(n) \mathbf{u}(n)-\left(d(n)-\mathbf{u}^{T}(n) \mathbf{w}(n)\right) \mathbf{u}^{T}(n-1) \mathbf{u}(n)}{\mathbf{u}^{T}(n) \mathbf{u}(n) \mathbf{u}^{T}(n-1) \mathbf{u}(n-1)-\left(\mathbf{u}^{T}(n) \mathbf{u}(n-1)\right)^{2}+\epsilon} .
\end{aligned}
$$

A1) $v_{i}(n)$ and $v_{o}(n)$ are zero-mean white noise with variance $\sigma_{i}^{2}$ and $\sigma_{o}^{2}$.

A2) $v_{i}(n)$ and $v_{o}(n)$ are independent and uncorrelated with $\mathbf{u}[n]$.

A3) The length $M$ of the FIR and the input noise variance $\sigma_{i}^{2}$ is a priori known.

\section{CONSISTENT BinORMALIZED DATA-REUSING LMS ALgORITHM}

The binormalized data-reusing LMS algorithm obtains the projections of two consecutive gradient directions. The statement of the problem is that to minimize the squared Euclidean norm of the change $\delta \mathbf{w}(n+1)=\mathbf{w}(n+1)-\mathbf{w}(n)$ subjected to $\quad \mathbf{u}^{T}(n) \mathbf{w}(n+1)=d(n) \quad$ and $\mathbf{u}^{T}(n-1) \mathbf{w}(n+1)=d(n-1)$. The cost function for the problem is, then.

$$
\begin{aligned}
J(n)= & \|\delta \mathbf{w}(n+1)\|^{2}+\lambda_{1}\left(d(n)-\mathbf{u}^{T}(n) \mathbf{w}(n+1)\right) \\
& +\lambda_{2}\left(d(n-1)-\mathbf{u}^{T}(n-1) \mathbf{w}(n+1)\right) .
\end{aligned}
$$

The problem has unique solution as

$$
\mathbf{w}(n+1)=\mathbf{w}(n)+\frac{\lambda_{1}}{2} \mathbf{u}(n)+\frac{\lambda_{2}}{2} \mathbf{u}(n-1)
$$

where $\lambda_{1}$ and $\lambda_{2}$ is given by (5)

However, we are only able to use a noisy measurement $\tilde{\mathbf{u}}(n)$ instead of $\mathbf{u}(n)$ when the input signal is noisy. Therefore, we exploit the following stochastic value with the assumptions (A1, A2):

$$
\begin{array}{ll}
E\left[\tilde{\mathbf{u}}(n) \tilde{\mathbf{u}}^{T}(n)\right] & =E\left[\mathbf{u}(n) \mathbf{u}^{T}(n)\right]+\sigma_{i}^{2} \mathbf{I} \\
E\left[\tilde{\mathbf{u}}^{T}(n) \tilde{\mathbf{u}}(n)\right] & =E\left[\mathbf{u}^{T}(n) \mathbf{u}(n)\right]+M \sigma_{i}^{2} \\
E\left[\tilde{\mathbf{u}}(n+1) \tilde{\mathbf{u}}^{T}(n)\right] & =E\left[\mathbf{u}(n+1) \mathbf{u}^{T}(n)\right] \\
E\left[\tilde{\mathbf{u}}^{T}(n+1) \tilde{\mathbf{u}}(n)\right] & =E\left[\mathbf{u}^{T}(n+1) \mathbf{u}(n)\right]
\end{array}
$$

Further, approximating the expectation value with instantaneous values, then we have

$$
\begin{array}{ll}
\mathbf{u}(n) \mathbf{u}^{T}(n) & \approx \tilde{\mathbf{u}}(n) \tilde{\mathbf{u}}^{T}(n)-\sigma_{i}^{2} \mathbf{I} \\
\mathbf{u}^{T}(n) \mathbf{u}(n) & \approx \tilde{\mathbf{u}}^{T}(n) \tilde{\mathbf{u}}(n)-M \sigma_{i}^{2} \\
\mathbf{u}(n+1) \mathbf{u}^{T}(n) & \approx \tilde{\mathbf{u}}(n+1) \tilde{\mathbf{u}}^{T}(n) \\
\mathbf{u}^{T}(n+1) \mathbf{u}(n) & \approx \tilde{\mathbf{u}}^{T}(n+1) \tilde{\mathbf{u}}(n)
\end{array}
$$

Substituting the $\lambda_{i}$ of (5) and the approximations of (7) into the update equation (4) and introducing scaling factor denoted by $\mu \in(0,1]$, we obtain

TABLE I: PROPOSED CONSISTENT BINORMALIZED DATA-REUSING LMS ALGORITHM

\begin{tabular}{|l|}
\hline Initialize: $\hat{\mathbf{w}}(1)=0$, \\
\hline For $n=1,2, \ldots$, \\
\hline$a=\tilde{\mathbf{u}}^{T}(n) \tilde{\mathbf{u}}(n)$ \\
$b=\tilde{\mathbf{u}}^{T}(n-1) \tilde{\mathbf{u}}(n-1)$ \\
$a=\tilde{\mathbf{u}}^{T}(n) \tilde{\mathbf{u}}(n-1)$ \\
$d=\tilde{\mathbf{u}}^{T}(n) \mathbf{w}(n)$ \\
$e=\tilde{\mathbf{u}}^{T}(n-1) \mathbf{w}(n)$ \\
$f=(M-1) \sigma_{i}^{2}$ \\
$e_{1}(n)=d(n)-\tilde{\mathbf{u}}(n)^{T} \hat{\mathbf{w}}(n)$ \\
$e_{2}(n)=d(n-1)-\tilde{\mathbf{u}}(n-1)^{T} \hat{\mathbf{w}}(n)$ \\
$A=e_{1}(b-f)-e_{2} c-\sigma_{i}^{2} d$ \\
$B=e_{2}(a-f)-e_{1} C-\sigma_{i}^{2} e$ \\
$C=\sigma_{i}^{2}(a+b-2 f)$ \\
$D=a b-c^{2}-M \sigma_{i}^{4}+\epsilon$ \\
$\hat{\mathbf{w}}(n+1)=\hat{\mathbf{w}}(n)+\mu(A \tilde{\mathbf{u}}(n)+B \tilde{\mathbf{u}}(n-1)+C \hat{\mathbf{w}}(n)) / D$ \\
\hline The step-size $\mu$ is pre-defined. $M$ denotes the length of the \\
unknown FIR and $\epsilon$ is small value \\
\hline
\end{tabular}

$\hat{\mathbf{w}}(n+1)=\hat{\mathbf{w}}(n)+\mu(A \mathbf{u}(n)+B \mathbf{u}(n-1)+C \hat{\mathbf{w}}(n)) / D$

where

$$
\begin{aligned}
A= & e_{1}(n)\left(\tilde{\mathbf{u}}^{T}(n-1) \tilde{\mathbf{u}}(n-1)-(M-1) \sigma_{i}^{2}\right) \\
& -e_{2}(n) \tilde{\mathbf{u}}^{T}(n) \tilde{\mathbf{u}}(n-1)-\sigma_{i}^{2} \tilde{\mathbf{u}}^{T}(n) \hat{\mathbf{w}}(n), \\
B= & e_{2}(n)\left(\tilde{\mathbf{u}}^{T}(n) \tilde{\mathbf{u}}(n)-(M-1) \sigma_{i}^{2}\right) \\
& -e_{1}(n) \tilde{\mathbf{u}}^{T}(n) \tilde{\mathbf{u}}(n-1)-\sigma_{i}^{2} \tilde{\mathbf{u}}^{T}(n-1) \hat{\mathbf{w}}(n), \\
C= & \sigma_{i}^{2}\left(\tilde{\mathbf{u}}^{T}(n-1) \tilde{\mathbf{u}}(n-1)+\tilde{\mathbf{u}}^{T}(n) \tilde{\mathbf{u}}(n)-2(M-1) \sigma_{i}^{2}\right), \\
D= & \tilde{\mathbf{u}}^{T}(n) \tilde{\mathbf{u}}(n) \tilde{\mathbf{u}}^{T}(n-1) \tilde{\mathbf{u}}(n-1)-\left(\tilde{\mathbf{u}}^{T}(n) \tilde{\mathbf{u}}(n-1)\right)^{2} \\
& -M \sigma_{i}^{4}+\epsilon, \\
& \quad e_{1}(n)=d(n)-\tilde{\mathbf{u}}(n)^{T} \hat{\mathbf{w}}(n), \\
& e_{2}(n)=d(n-1)-\tilde{\mathbf{u}}(n-1)^{T} \hat{\mathbf{w}}(n) .
\end{aligned}
$$

And

The proposed algorithm is summarized in Table I

\section{EXPERIMENTAL RESULTS}

In order to compare the performance of the proposed algorithm with other adaptive filtering algorithms, we carried the following FIR identification experiment. The FIR of the unknown system is generated by randomly with the length of 
16. The input signal $u(n)$ is generated as a white, zero-mean, Gaussian random sequence or obtained by filtering it through a first-order system $G_{1}(z)=1 /\left(1-0.8 z^{-1}\right)$.

The signal to noise ratio (SNR) of the input is set to $20 \mathrm{~dB}$ which is defined by and the SNR of the measurement is set to $30 \mathrm{~dB}$ which is defined by

$$
\begin{array}{r}
\mathrm{SNR}_{i}=10 \log _{10} \sum_{n} u(n)^{2} / \sum_{n} v_{i}(n)^{2}, \\
\mathrm{SNR}_{o}=10 \log _{10} \sum_{n}\left(\tilde{\mathbf{u}}^{T}(n) \mathbf{h}\right)^{2} / \sum_{n} v_{o}(n)^{2}
\end{array}
$$

The estimation error as the performance measure is obtained by using the mean square deviation (MSD) defined as $\operatorname{MSD}(n)=(\mathbf{h}-\hat{\mathbf{w}}(n))^{T}(\mathbf{h}-\hat{\mathbf{w}}(n)) / \mathbf{h}^{T} \mathbf{h} \quad$ and the simulation results are obtained by ensemble averaging over 100 independent trials.

We tested both a white input sequence and correlated input sequence and the compared performance of the proposed algorithm is illustrated in Fig. 1 and Fig 2, respectively. We compared the proposed algorithm with several related studies of the NLMS, the consistent NLMS (C-NNLS), and BNDR-LMS [7], [8]. As expected the performance of the proposed algorithm is very similar with that of the C-NLMS when we use a white input sequence and they are better then the performance of NLMS and BNDR-LMS Fig 1.

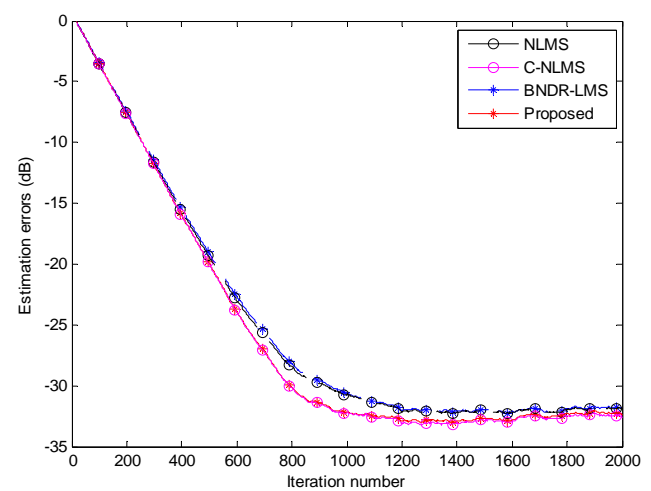

Fig. 1. Mean-square deviation of NLMS, C-NLMS, BNDR-LMS, and the proposed algorithm using white input sequences

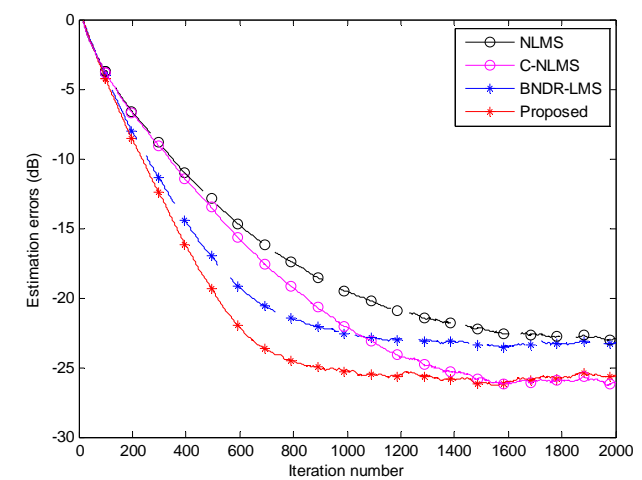

Fig. 2. Mean-square deviation of NLMS, C-NLMS, BNDR-LMS, and the proposed algorithm using correlated input sequences

However, when the colored input which is generated by first order AR model was used, the proposed algorithm achieved fast convergence speed compared to C-NLMS while having smaller steady-state estimation errors than that of NLMS and BNDR_LMS (Fig 2).

\section{CONCLUSION}

A consistent binormalized data-reusing least mean square (LMS) algorithm for identifying FIR models whose input and output are corrupted by additive white noise was proposed. By exploiting the stochastic properties of the noisy input, the proposed algorithm compensated a bias of estimation. Moreover, in order to improve convergence performance, the input data was reused. The simulation results of an adaptive FIR estimation showed that the proposed algorithm achieved consistent estimation with noisy input signal. Furthermore, the proposed algorithm had faster convergence rate than the ordinary consistent LMS algorithms while having smaller steady-state estimation errors than that of NLMS and BNDR_LMS when the input signal was highly correlated.

\section{REFERENCES}

[1] S. Haykin, Adaptiv Filter Theory, Englewood Cliffs, NJ: Prentice-Hall, 1996

[2] G. H. Golub and C. F. Van Loan, "An analysis of the total least squares problem,” in SIAM Number. Anal., vol. 17, Dec. 1980, pp. 883-893

[3] P. Stoica and T. Soderstrom, "Bias correction in least-squares identification,” Int. J. Control, vol. 35, pp. 449457, 1982

[4] D. Roberto, "A bias-compensated identification approach for noisy FIR Models," IEEE Trans. Signal Processing letters, vol. 15, pp. 325-328, 2008

[5] C. E. Davila, "An efficient recursive total least squares algorithm for FIR adaptive filtering," IEEE Trans. Signal Processing, vol. 42, pp. 268-280, Feb. 1994

[6] D. Z. Feng, X. D. Zhang, D. X. Chang, and W. X. Zheng, "A Fast Recursive Total Least Squares Algorithm for Adaptive FIR Filtering," IEEE Trans. Signal Processing, vol. 52, pp. 2729-2737, Oct. 2004

[7] S. Jo, "Consistent normalized least mean square filtering with noisy data matrix," IEEE Trans. Signal Processing, vol. 53, pp. 2112-2123, June. 2005

[8] J. A. Apolinario, M. L. R. D. Campos, and P. S. R. Diniz, "The binormalized data-reusing LMS algorithm," in Proc. of XV Simposio Brasileiro de Telecomunicaoes, Recife, Brazil, 1997, pp. 7780

[9] J. A. Apolinario, M. L. R. D. Campos, and P. S. R. Diniz, "Convergence analysis of the binormalized data-reusing LMS algorithm,” IEEE Trans. Signal Processing, vol. 48, pp. 3235-3243, Nov. 2000

Byung Hoon Kang received his BS in electrical engineering from Pohang University of Science and Technology (POSTECH) in 2005. He is currently studying toward a $\mathrm{PhD}$ at POSTECH. His current research interests are image processing and signal processing.

Nam Gyu Kwon received his BS in electrical engineering from Pohang University of Science and Technology (POSTECH) in 2010. He is currently studying toward a MS at POSTECH. His current research interests are image processing and signal processing.

Hyon-Taek Choi received the BS, MS, and $\mathrm{PhD}$ in Electronic Engineering from Hanyang University of Seoul, Korea. in 1991, 1993 and 2000, respectively. From 1993 to 1995, he was a Researcher in the Multimedia Laboratory of Korea Telecommunication. From 2000 to 2003, he was a Post-Doctoral Fellow in the Autonomous Systems Laboratory at the University of Hawaii at Manoa. He is currently a Principal Researcher in the Korea Ocean Research \& Development Institute. His current research interests include robust and optimal control, embedded system, navigation system, sensor fusion, and artificial intelligence for underwater robotic vehicles.

Poo Gyeon Park received his BS and MS in control and instrumentation engineering from Seoul National University, Korea, in 1988 and 1990, respectively, and his $\mathrm{PhD}$ in electrical engineering from Stanford University, in 1995. Since 1996, he has been affiliated with the Division of Electrical and Computer Engineering at Pohang University of Science and Technology, where he is currently a professor. His current research interests include robust, Linear Parameter Varying (LPV), Receding Horizon Control (RHC), intelligent, and network-related control theories, signal processing, and wireless communications for a personal area network. 